\title{
Continuous attention: Rationale and discriminant validation of a test designed for use in psychopharmacology
}

\author{
BRIAN TIPLADY \\ Royal Infirmary, Edinburgh, Scotland
}

\begin{abstract}
The continuous attention task (CAT) is a test designed to assess changes in attention due to a variety of factors, for example, drugs. Subjects view a series of $3 \times 3$ patterns of squares on a monitor screen, each displayed for $100 \mathrm{msec}$ at intervals of 1.5-2.5 sec, and respond whenever two successive patterns are identical. For such a measure to be valid, factors other than attention should be investigated, and shown not to be a factor in performance. Nineteen subjects took part in a study in which information-processing rate and recall of CAT figures was measured. The results showed that a viewing time of 50-60 msec was sufficient for 50\% correct recognition of CAT figures, and that recognition with a masked presentation of $100 \mathrm{msec}$ did not differ significantly from an unmasked presentation of $100 \mathrm{msec}$. Mean recall of CAT figures $2 \mathrm{sec}$ after a 100-msec exposure was $98.2 \%$. These results suggest that performance on the CAT is not limited by either informationprocessing speed or memory capacity, but is a valid measure of the ability to sustain attention.
\end{abstract}

Attention is of great importance in human performance, as the ability to select information from the environment and to sustain efficient input is often a major constraint. Many factors affect performance on tasks designed to assess attention or vigilance, and these are frequently associated with changes in arousal. Examples of such effects are those of sleep deprivation, noise, and drugs.

The effects of drugs on performance have been the subject of much concern, particularly with regard to their potential to contribute to accidents, whether on the road, at work, or at home. Sedative drugs such as pentobarbital, chlorpromazine, diazepam, mianserin, and nitrous oxide produce impairments in attention-related tasks, whereas stimulants such as caffeine and amphetamine lead to improvements (Clubley, Bye, Henson, Peck, \& Riddington, 1979; Fagan, Paul, Drummond, Scott, \& Tiplady, 1989; Fagan, C. G. Swift, \& Tiplady, 1988; Hart, Hill, Bye, Wilkinson, \& Peck, 1976; Mackworth, 1965; Mirsky \& Kornetsky, 1964; C. G. Swift, M. R. Swift, \& Tiplady, 1988).

The nature of the performance tasks used must be considered if these changes are to be convincingly interpreted in terms of attention. Drugs may affect many aspects of human abilities, and it is important that valid tests be used both so that results may be interpreted correctly and so that a test battery can be designed that will address a broad range of functioning in order to obtain a profile of a drug's effects. Two factors are of importance in attention tasks,

The continuous attention task (CAT) is available for both BBC- and IBM-compatible microcomputers. Inquiries should be addressed to the author. Correspondence and requests for reprints should be addressed to Brian Tiplady, Astra Clinical Research Unit, 10 York Place, Edinburgh EH 1 3EP, U.K. the first being the discriminability of the stimulus and the second, automaticity.

In some tasks, the stimulus used is close to the threshold for discriminability. This is the case with the auditory vigilance task (Wilkinson, 1968), where the subject must discriminate the duration of a tone against a background of white noise. Typically, the subject will detect only about $50 \%$ of the target (shorter) tones. For such tests there is a possibility that perceptual factors could impair performance without there being any change in attention itself. For example, local anesthetic drugs can produce tinnitus (Descotes \& Evreux, 1988), an effect clearly likely to lead to impairment on a near-threshold auditory task. In other tasks, such as the continuous performance task (Rosvold, Mirsky, Sarason, Bransome, \& Beck, 1956) and the continuous attention task (Tiplady, 1985, 1988), which is the subject of the present paper, stimuli (letters and geometric patterns) are readily discriminable. Failure to detect a stimulus cannot, in this case, be due to perceptual difficulties, but is attributed rather to failure to maintain an alert state.

A second factor is whether the processing is automatic or controlled (Schneider \& Shiffrin, 1977). These two types of processing are probably best thought of as extremes of a continuum. Simple, overlearned tasks will be predominantly automatic. Some tasks, for example those requiring simultaneous analysis of a number of features, are always effortful. Many tasks start as effortful and become increasingly automatic with practice. In psychopharmacological testing, both automatic and control processes need to be investigated if a complete profile of drug effects is to be obtained, although it has been suggested that automatic processing is much less likely to be affected by state changes than is effortful processing (Hasher \& Zachs, 1979). Problems of interpretation are likely to $\alpha$ - 
cur, however, with a test that is automated to an uncertain, and possibly changing, degree. This may be the case with the continuous performance test, originally devised to assess brain damage, but also often used for studying drug effects (see, e.g., Mirsky \& Rosvold, 1960). In the continuous performance test, subjects are presented with a series of letters presented on a monitor screen and are instructed to respond when a particular letter (usually an $\mathrm{X}$ ) or sequence of letters (usually $\mathrm{A}_{-} \mathrm{X}_{\text {) }}$ appears. Letter recognition and matching are processes clearly capable of being performed automatically.

The continuous attention task (CAT) was designed for use in pharmacology, although, like the continuous performance task, it may be suitable for assessing attention in other contexts (Tiplady, 1985, 1988). In this task, the stimuli are geometrical shapes flashed for $100 \mathrm{msec}$ (Figure 1). This choice of material was intended to avoid familiar, easily automated stimuli. The display time was chosen as being sufficient to allow for easy perception of the stimulus, while being substantially shorter than the saccadic reaction time of about $200 \mathrm{msec}$ (see, e.g., Fischer \& Breitmeyer, 1987). The task also differs from the continuous performance test in that the subject responds not to a particular target shape, but to the same shape's appearing twice in succession. This introduces a memory load into the task, a factor that has been shown to be important in the occurrence of performance decline over time in sustained attention tasks (Parasuraman, 1979).

At about the same time as the CAT was being developed, Cornblatt, Risch, Faris, Friedman, and ErlenmeyerKimling (1988) made a similar modification to the continuous performance test, using the repetition of the stimulus letter rather than a fixed letter or sequence of letters as the target (the identical pairs version, CPT-IP). A further difference between the CAT and the continuous performance test is that the intervals between stimuli in the former are not constant, but are varied randomly between 1.5 and $2.5 \mathrm{sec}$. This increases the attentional requirement by preventing the subjects from pacing their alertness to the rhythm of the task.

While performance on the CAT clearly requires sustained attention, it is necessary to consider whether other factors might be involved. The most obvious of these is memory. Correct recognition of the repeated stimulus depends upon visual memory, as well as upon attention. If this memory requirement is a limiting factor, drug-induced memory impairments, which occur with many psychotropic drugs (see, e.g., Preston et al., 1989), could be the source of decrements in test performance. This would invalidate the test and lead to incorrect conclusions concerning drug action.

When the CAT was designed, the memory load was chosen so as to be well within the presumed capacity of visual memory, and thus not likely to be a limiting factor. However, in view of the limited amount of work on visual memory, it was considered desirable to investigate memory processes involved with processing CAT figures, to test whether this was indeed the case. This enabled a form of discriminant validation to be carried out on the CAT. That is, a valid test should be affected by the variables it is supposed to be measuring, but not by other variables that it is not supposed to be measuring (after Anastasi, 1988, pp. 156-157).

The two aspects of memory most likely to place limitations on performance are the rate of transfer of information from the perceptual memory store (iconic memory) and the capacity of the short-term store. The capacity of iconic memory is much larger than that of the shortterm store, and should not be limiting (Sperling, 1960). Given that the response in the CAT is made only a few seconds after presentation of the stimulus, long-term memory should not be limiting. The final stage of the test is the production of the response. This is simple, and not timed, and is unlikely to be a limiting factor.

In the present study, perception and memory capacity for CAT figures were investigated. The viewing time necessary for accurate perception was studied using a backwardmasking paradigm with forced-choice responses. If viewing times of less than $100 \mathrm{msec}$ are sufficient for accurate and reliable perception of $3 \times 3$ figures, then it may be concluded that the rate of transfer from iconic memory is not limiting CAT performance. Unmasked presentations were also used, followed by both forced-choice and free-recall responses. Accurate recall of $3 \times 3$ figures after unmasked presentation would indicate that the capacity of the short-term store was nor limiting CAT performance.

A further measure was included in the study for comparison-inspection time (Vickers, Nettelbeck, \& Willson, 1972). This is a measure of central processing speed in which a different discrimination is used which involves a much smaller amount of information (which of two lines is longer) than is the case with the $3 \times 3$ figures. The hypothesis being tested here did not relate to the validity of the CAT, but to the question of whether two rather similar tests involving very different amounts of information would give comparable results.

The aims of the study were thus (1) to examine the CAT to determine whether it could be considered a valid measure of attention, and (2) to compare it with a simpler twochoice procedure.

\section{METHOD}

\section{Subjects}

Twenty subjects took part in the study. Due to a technical failure, data from 1 subject was incomplete and was not included in the analysis. The remaining subjects were 6 males and 13 females, aged 20-38.

\section{Test Procedures}

After a session in which all the tests were given for purposes of familiarization, the subjects took part in a $11 / 2-h$ test session. All tests were presented, and responses recorded, on a BBC Master 128 microcomputer. The monitor was a CUB 1451MS4 (Microvitec, Ltd., Bradford, UK; refresh rate, $50 \mathrm{~Hz}$; phosphor decay time to $10 \%$, 


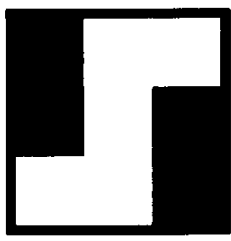

(a)

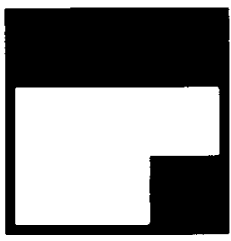

(c)

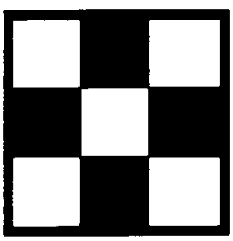

(b)

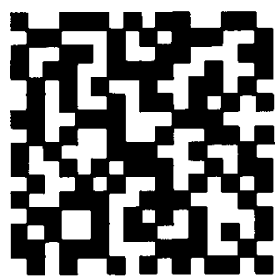

(d)
Figure 1. (a-c) Examples of stimulus figures from the continuous attention task. Black and white are reversed, as the figures are presented on a dark monitor screen. Patterns are flashed onto the screen for 100 msec at intervals of 1.5-2.5 sec. The subject's task is to press a button whenever two consecutive patterns are the same. (d) The mask used in the viewing time experiments.

$<1 \mathrm{msec}$ ). The tests, given in randomized order, were the following:

Continuous attention task (CAT, Tiplady, 1988). Each stimulus consisted of a $3 \times 3$ pattern of four light and five dark squares (Figure 1) presented for $100 \mathrm{msec}$, with a randomized interstimulus interval of between 1.5 and $2.5 \mathrm{sec}$. The subject responded by pressing a button whenever two successive patterns were the same. Responses were not timed, and the subject had $1 \mathrm{sec}$ in which to make a response. A total of 241 presentations, with 40 repetitions, was used. Presentations were in blocks of 6, with one repetition at a random position within each block. This blocking was used in order to spread the target stimuli relatively evenly through the test period. The block structure was not apparent to the subject. Successive nontarget patterns differed by the interchange of one light and one dark square, selected at random. The measures obtained were the total number of correct responses $(C)$; the total number of incorrect responses $(I)$; and the error index, $(40-C) / 40+2(I / 200)$, as described by Pigache (1976). This composite index was designed to reflect the overall severity of impairment to performance.

Pattern recall. The subjects were presented with a series of randomly generated CAT stimuli on the monitor screen, each being displayed for $100 \mathrm{msec}$. After each stimulus, a 2-sec period elapsed, after which the subject attempted to reproduce the pattern by marking a printed $3 \times 3$ frame with the positions of the light squares. After making the response, the subject pressed a button for the next stimulus. Forty-eight stimuli were presented, and the last 40 were analyzed. The number of correct responses out of $\mathbf{4 0}$ was recorded.

Information transfer rate, autopresentation. The subjects were presented with a randomly generated sequence of CAT figures. Each figure was followed by a mask consisting of a random pattern of small black and white squares (Figure 1d). The mask was displayed for $1 \mathrm{sec}$. The interval between the onset of the stimulus and that of the mask (viewing time) was $20,40,60,80$, or $100 \mathrm{msec}$. An unmasked condition with an exposure time of $100 \mathrm{msec}$ was also used. Four test patterns then appeared on the screen; one of these was the stimulus figure previously presented, and the others were different CAT patterns. The subject pressed one of four buttons to indicate the recognized pattern. The subsequent stimulus pattern appeared after a randomized interval of 1.5-2.5 sec. Twelve blocks of 22 trials were presented, each block consisting of a single masking condition. The subjects were presented with one series of six blocks with decreasing viewing time, beginning with the unmasked condition, and one series of six blocks with increasing viewing time, ending with the unmasked condition, in balanced fashion. The first two stimuli in each block were for familiarization, and were not analyzed. Thus, 40 responses were analyzed at each viewing time.

Information transfer rate, self-paced. The above procedure was repeated, except that after each trial the subject pressed the response button a second time to initiate the next stimulus. This was presented after a delay of $1.2 \mathrm{sec}$. This interval was chosen from pilot studies to match the average presentation rates in the two conditions.

Inspection time (Vickers et al., 1972). In this measure of processing speed, the subject was presented with two illuminated vertical lines, one $24 \mathrm{~mm}$ long and the other $48 \mathrm{~mm}$ long. The lines were $30 \mathrm{~mm}$ apart. This was followed by a mask consisting of two longer lines, each $60 \mathrm{~mm}$ long (Figure 2). The subject responded by press-

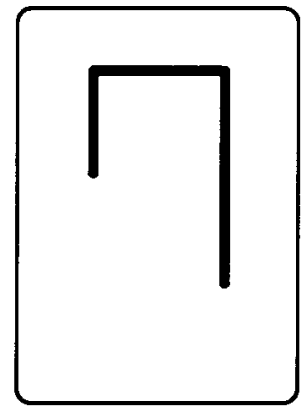

(a)

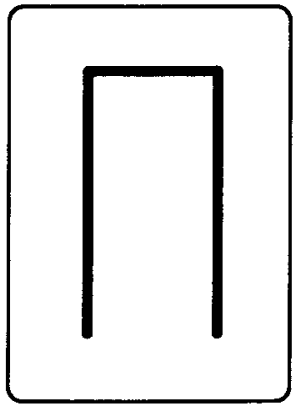

(b)
Figure 2. Inspection time. A display with two vertical lines, one longer than the other, is illuminated (a). After an interval (the interstimulus interval), the stimulus is followed by a mask (b). The short and long lines are 24 and $48 \mathrm{~mm}$ long; the masking lines are $60 \mathrm{~mm}$ long. The subject sits $1 \mathrm{~m}$ in front of the display. The subject presses a left or right button to indicate the longer line. 
ing a left or right button, to indicate the longer line. The viewing time (or inspection time) was varied according to the PEST aigorithm, an adaptive procedure which decreases viewing time when subjects perform accurately and increases it when errors occur. The step size gradually decreases, so that the algorithm "homes in" to the inspection time necessary for $85 \%$ correct recognition (IT85). The number of trials is not fixed. The algorithm uses as many trials as necessary to obtain a sufficiently accurate estimate of IT85 (Taylor \& Creelman, 1967).

\section{Statistical Analysis}

For each subject, the recognition scores for the masked presentations were analyzed using probit analysis with .25 (chance performance level) as the natural response rate (Lieberman, 1983; SAS Institute, 1989). This method fits a sigmoid cumulative normal curve to the data from each subject, and gives two parameters, mu and sigma, respectively the mean and standard deviation of the resulting normal curve. Mu represents an estimate of the viewing time necessary for $62.5 \%$ correct responses, this being the midpoint between minimum performance (chance level, $25 \%$ ) and maximum performance (100\%). The values of these parameter estimates were compared between autoand self-paced presentation using Student's $t$ test (paired). Correlations between the values of mu, sigma, and the

Table 1

Main Summary Statistics

\begin{tabular}{|c|c|c|c|c|c|}
\hline Measure & Mean & $S D$ & Min & Max & $p$ \\
\hline \multicolumn{6}{|c|}{ Continuous Attention } \\
\hline$N$ correct $(\max 40)$ & 36.8 & 3.0 & 29 & 40 & - \\
\hline$N$ incorrect $(\max 200)$ & 1.1 & 1.2 & 0 & 4 & - \\
\hline Error index & 0.091 & 0.080 & 0.000 & 0.295 & - \\
\hline \multicolumn{6}{|c|}{ Pattern Recall } \\
\hline$N$ correct $(\max 40)$ & 39.3 & 0.87 & 38 & 40 & - \\
\hline \multicolumn{6}{|c|}{ Information Transfer Rate-Self-paced } \\
\hline Mu (msec) & 52 & 11 & 37 & 86 & - \\
\hline Sigma (msec) & 19 & 6.4 & 7 & 29 & - \\
\hline N correct, $100 \mathrm{msec}$ & 38.4 & 1.5 & 36 & 40 & - \\
\hline $\mathrm{N}$ correct, unmasked & 38.8 & 0.99 & 37 & 40 & - \\
\hline \multicolumn{6}{|c|}{ Information Transfer Rate-Autopaced } \\
\hline Mu (msec) & 58 & 9.0 & 47 & 80 & $<.01^{*}$ \\
\hline Sigma (msec) & 17 & 3.5 & 12 & 27 & n.s.* \\
\hline$N$ correct, $100 \mathrm{msec}$ & 38.8 & 1.7 & 33 & 40 & n.s. $\dagger$ \\
\hline N correct, unmasked & 38.9 & 1.0 & 37 & 40 & n.s. \\
\hline \multicolumn{6}{|c|}{ Inspection Time } \\
\hline IT85 (msec) & 41 & 13 & 22 & 84 & - \\
\hline
\end{tabular}

Note-Mu and sigma are, respectively, the mean and standard deviation of the cumulative normal distribution fitted to the recognition response curve by probit analysis (SAS Institute, 1989). IT85 is the viewing time necessary for $85 \%$ correct response in the inspection time procedure. $\quad$ *Statistical significance was tested for mu and sigma between the auto- and self-paced conditions (paired $t$ test). †Statistical significance was tested between the auto- and self-paced conditions. In each case, the mean of the unmasked and $100 \mathrm{msec}$ was taken (Wilcoxon signed ranks test). \$Statistical significance was tested between the 100 -msec and unmasked presentations. In each case, the mean of the auto- and self-paced conditions was taken (Wilcoxon signed ranks test).

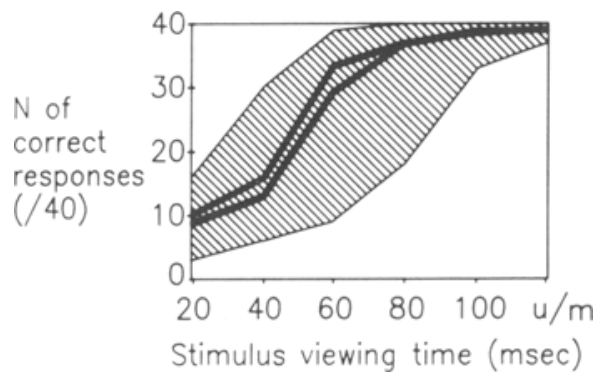

Figure 3. Recognition of continuous ath ution takk figures with backward masking. Forty stimull were presented at each viewing time. The mean number of correct responses at each time are shown for self-paced (upper bold line) and autopaced (lower bold line) conditions, together with the composite range of individual responses (hatched area).

error index for the CAT (after square-root transformation, since this measure is positively skewed) and IT85 were obtained using Pearson's coefficient. Scores for correct responses on the 100 -msec and unmasked exposures of the information transfer experiments were compared using the Wilcoxon signed-ranks test.

\section{RESULTS}

Results from the main statistical analysis are shown in Table 1. Data from the two masking conditions (auto- and self-paced) show that a shorter interval between stimulus and mask is required for given accuracy with self-pacing [mu $=52.2 \mathrm{msec}$ for self-pacing and $57.7 \mathrm{msec}$ for autopacing; $t(18)=3.14, p<.01]$. This is also illustrated in Figure 3. Of the 19 subjects, 13 showed greater values of mu for the autopaced condition. There was no statistically significant difference between the estimated values of sigma for the two conditions.

When results for the number of correct responses at the maximum viewing time in the masked $(100 \mathrm{msec})$ and unmasked conditions were compared, no significant differences were found either for the effects of masking or for the effects of auto- versus self-pacing. In all the unmasked conditions, the rates of correct responses were high, varying from $96.1 \%$ (recognition, $100 \mathrm{msec}$, self-paced) to $98.2 \%$ (recall).

The correlation matrix for the principal test measures is shown in Table 2. The only significant correlation is that between the values of mu determined from the autoand self-paced conditions. Correlations approaching significance are found for sigma determined from the two pacing conditions (.42) and between inspection time and the error index (.38). All other correlations are low $(<.3)$.

\section{DISCUSSION}

The values for mu and sigma obtained in the masking experiment indicate that the display time of $100 \mathrm{msec}$ used in the CAT is located at over $2 S D$ s above the mean of the probit curve. This duration should be sufficient for complete transfer of the pattern information from iconic 
Table 2

Correlation Matrix for Test Measures

\begin{tabular}{lrrrrr}
\hline & Mu-S & Si-A & Si-S & EI & IT85 \\
\hline Mu-A (Mu autopaced) & $.75^{*}$ & -.17 & .02 & -.10 & .21 \\
Mu-S (Mu self-paced) & & -.14 & -.18 & -.02 & .09 \\
Si-A (Sigma autopaced) & & & .42 & .19 & -.15 \\
Si-S (Sigma self-paced) & & & & .26 & .08 \\
CAT error index & & & & & .38
\end{tabular}

Note-Tabulations are of Pearson's coefficient $r$. CAT $=$ continuous attention task. Mu and sigma are, respectively, the mean and standard deviation of the cumulative normal distribution fitted to the recognition response curve by probit analysis (SAS Institute, 1989). ${ }^{*}|r|$ significantly greater than $0, p<.01$.

memory. This conclusion is supported by the high proportion of correct responses in the 100 -msec responses (>96\%), and by the fact that the proportion of correct responses for the unmasked condition is no higher than that for the $100-\mathrm{msec}$ masked condition. Thus, the time available for inspection of the patterns in the CAT (which corresponds to the unmasked condition in the recognition tests) should be more than sufficient.

Several previous studies have addressed issues related to those raised here, though for rather different reasons. For example, Moskowitz and Murray (1976a, 1976b) used a backward-masking paradigm to investigate the effects of alcohol on information transfer. Their stimuli were sets of four letters followed, after an interval of $15-75 \mathrm{msec}$, by a mask consisting of jumbled up bits of letters. They showed that with an interval of $55 \mathrm{msec}$ between onset of stimulus and onset of mask there was about $50 \%$ correct recall of the stimulus letters. They were also able to show decrements in transfer rate with ethanol.

Inui (1988) has studied a series of block patterns of different sizes using masking. This work suggested that for a $3 \times 3$ pattern, a viewing time between 100 and $280 \mathrm{msec}$ is necessary for optimal performance. However, few time points were assessed and no unmasked condition was used.

It is interesting that the CAT patterns required about the same time for processing as did the letters used by Moskowitz and Murray (1976a, Figure 1). A viewing time of about $55 \mathrm{msec}$ was required in both cases, although the CAT patterns contained less information than the letters (about 7 bits for a $3 \times 3$ block pattern, since there are 126 possible patterns, as opposed to about 19 bits for four letters). This could indicate that processing of nonverbal information is slower than that of letters, or that partial recall was possible in the letter experiment, whereas the present paradigm required a completely correct response.

The inspection time measure was included in this analysis because it is an established procedure which is similar to the block pattern task in using backward masking followed by a forced-choice response. The lack of correlation between inspection time and mu in the present study suggests that in spite of these similarities, the two tests measure different things. However, whether the important factor is the difference in the amount of information processed ( 1 bit for inspection time, since there are only two possible stimuli) or is the type of discrimination being made cannot be assessed from the present experiments. Also, the number of subjects in the present study is rather small for obtaining reliable correlations, and it would be desirable to extend this aspect of the work.

The recall of CAT patterns appears to be at least as good as their recognition. The percentage of correct recalls (98.2\%) compares well to that of $96.7 \%$ for recognition of $4 \times 4$ patterns reported by Phillips and Christie (1977). Taken together, these results indicate that STS capacity should be more than adequate for CAT figures. Thus, visual short-term memory is unlikely to be a constraint on performance of the CAT.

The difference between the auto- and self-paced conditions may be taken as an indication of the effects of attentional processes, since attention is likely to be better concentrated when the subject controls the presentation of the stimuli. In the self-paced condition, the stimulus appeared at a fixed time after the subject pressed a button to initiate the presentation. In the autopaced condition, the stimulus appeared at an interval that varied randomly between 1.5 and $2.5 \mathrm{sec}$ after the preceding response, preventing the subject from pacing his attention in a regular fashion in a manner similar to that used in the CAT itself. It is interesting that this difference is reflected in the viewing time, as determined by mu, but not in the asymptote, that is, the performance at $100 \mathrm{msec}$ or unmasked. The CAT corresponds to performance in the unmasked condition. This suggests the possibility that different aspects of attention are involved in the two measures, a suggestion that is supported by the finding that the error index derived from the CAT does not show any marked correlation with mu (Table 2).

These two different parameters correspond to the different ways in which attention has been regarded in the literature. According to one approach, attention is regarded as a processing capacity that can be allocated to different sources of input (see, e.g., Wickens, 1984). On this view, a decrement in attention is seen as a reduction in processing capacity. Thus, the increase in time necessary for processing figures in the autopaced condition, as well as that reported for alcohol as mentioned above, may be interpreted as a reduction in the availability of a processing resource under these conditions. An alternative view emphasizes the importance of the maintenance of an alert state, that is, the capacity to register information at all. This approach is related to the concept of "blocking," where intermittent, more or less complete failures of attention occur (Bills, 1931; Valley \& Broughton, 1983). Failures to detect stimuli on the normal CAT or continuous performance test paradigm would appear to correspond more closely to this kind of lapse. These refractory periods, also referred to as microsleeps or involuntary rest pauses (Bjerner, 1949; Eysenck \& Frith, 1977; Morris, Williams, \& Lubin, 1960), may be of particular relevance to studies involving driving, in view of the frequency of reports from drivers involved in accidents in which they had "looked but did not see" (Sabey \& Taylor, 1980). 
It would be of interest to investigate the differential effects of drugs on these two measures-mu, representing a processing resource, and asymptotic performance, indicating sustained alertness. If dissociation between different drugs in terms of different attentional measures could be shown, it would be of great importance for the interpretation of the actions of different classes of drug.

In conclusion, these results support the suggestion that performance on the CAT is not limited either by information-processing speed or by memory capacity, but is a measure of the ability to sustain attention on a single source of information.

\section{REFERENCES}

Anastasi, A. (1988). Psychological testing (6th ed.). New York: Macmillan.

BiLLs, A. G. (1931). Blocking-a new principle of mental fatigue. American Joumal of Psychology, 43, 230-245.

BjerNer, B. (1949). Alpha depression and lowered pulse rate during delayed actions in a serial reaction test. Acta Physiologica Scandinavica, 19(Suppl. 65), 1-93.

Clubley, M., Bye, C. E., Henson, T. A., Peck, A. W., RiddingTON, C. J. (1979). Effects of caffeine and cyclizine alone and in combination on human performance, subjective effects and EEG activity. British Journal of Clinical Pharmacology, 7, 157-163.

Cornblatt, B. A., Risch, N. J., Faris, G., Friedman, D., ERLENMEYER-KIMLING, L. (1988). The continuous performance test, identical pairs version (CPT-IP). 1. New findings about sustained attention in normal families. Psychiatry Research, 26, 223-238.

Descotes, J., EvreuX, J.-C. (1988). In M. N. G. Dukes (Ed.), Meyler's side effects of drugs (pp. 217-223). Amsterdam: Elsevier.

Eysenck, H. J., \& FrTH, C. D. (1977). Reminiscence, motivation, and personality: A case study in experimental psychology. New York: Plenum.

Fagan, D., Paul, D., Drummond, G., Scott, D. B., \& TiPlady. B. (1989). A dose-response study of the effects of nitrous oxide on mood and performance in healthy volunteers. Journal of Psychopharmacology, 3, 98P

Fagan, D., Swift, C. G., \& TIPLADY, B. (1988). Effects of caffeine on vigilance and other performance tests in normal subjects. Journal of Psychopharmacology, 2, 19-25.

FisCHER, B., \& BREITMEYER, B. (1987). Mechanisms of visual attention revealed by saccadic eye movements. Neurophysiologia, 25, 73-83.

Hart, J., Hill, H. M., Bye, C. E., Wilkinson, R. T., \& PeCk, A. W. (1976). The effects of low doses of amylobarbitone sodium and diazepam on human performance. British Journal of Clinical Pharmacology, 3, 289-298.

HASHER, L., \& ZACKS, R. T. (1979). Automatic and effortful processes in memory. Journal of Experimental Psychology: General, 108, 356-388.

INUI, T. (1988). Properties of human visual memory for block patterns. Biological Cybernetics, 59, 179-187.

Lieberman, H. R. (1983). Computation of psychophysical thresholds using the probit technique. Behavior Research Methods \& Instrumentation, 15, 446-448.

MackworTH, J. F. (1965). The effect of amphetamine on the detectability of signals in a vigilance task. Canadian Journal of Psychology, 19, 104-111.

Mirsky, A. F., \& Kornetsky, C. (1964). On the dissimilar effects of drugs on the digit-symbol substitution and continuous performance tests. Psychopharmacologia, 5, 161-177.
Mirsky. A. F., Rosvold, H. E. (1960). The use of psychoactive drugs as a neuropsychological tool in studies of attention in man. In L. Uhr \& J. G. Miller (Eds.), Drugs and behavior (pp. 375-392). New York: Wiley.

Morris, G. O., Williams, H. L., \& LUBIN, A. (1960). Misperception and disorientation during sleep deprivation. Archives of General Psychiatry, 2, 247-254.

Moskowitz, H., MurRay, J. T. (1976a). Alcohol and backward masking of visual information. Joumal of Studies on Alcohol, 37 , 40-45.

Moskowitz, H., Murray, J. T. (1976). Decrease of iconic memory after alcohol. Journal of Studies on Alcohol, 37, 278-283.

Parasuraman, R. (1979). Memory load and event rate control sensitivity decrements in sustained attention. Science, 205, 924-927.

Phillips, W. A., Chrostie, D. F. M. (1977). Components of visual memory. Quarterly Joumal of Experimental Psychology, 9, 117-133.

Pigache, R. M. (1976). Comparison of scoring methods for tests of attention, including an error index for use with schizophrenic patients. Perceptual \& Motor Skills, 42, 243-253.

Preston, G. C., Ward, C., lines, C. R., Poppleton, P., Haigh, J. R. M., Traub, M. (1989). Scopolamine and benzodiazepine models of dementia: Cross-reversals by Ro 15-1788 and physostygmine. Psychopharmacology, 98, 487-494.

Rosvold, H. E., Mirsky, A. F., SArason, I., Bransome, E. D., Jr., - BeCK, L. H. (1956). A continuous performance test of brain damage. Journal of Consulting Psychology, 20, 343-350.

SABEY, B. E., TAYLOR, H. (1980). The known risks we run: The highway. In R. C. Schwing \& W. A. Abers, Jr. (Eds.), Societal risk assessment (pp. 43-70). New York: Plenum.

SAS INSTITUTE INC. (1989). SAS/STAT user's guide (Version 6, Vol. 2, 4th ed.). Cary, NC: SAS Institute.

SChNeIDER, W., ShIFFrIn, R. M. (1977). Controlled and automatic human information processing. Psychological Review, 1, 1-66.

SPERLING, G. (1960). The information available in brief presentations. Psychological Monographs, 74, 1-29.

Swift, C. G., SWifT, M. R., TIPlady, B. (1988). "First-dose" response to mianserin: Effects of age. Psychopharmacology, 96, 273-276.

Taylor, M. M., Creelman, C. D. (1967). Pest: Efficient estimates on probability functions. Journal of the Acoustical Society of America, 41, 782-787.

TIPLADY, B. (1985). An automated test battery for the detection of changes in mental function and psychomotor performance. British Jourmal of Clinical Pharmacology, 20, 305P.

TIPLADY, B. (1988). A continuous attention test for the assessment of the acute behavioral effects of drugs. Psychopharmacology Bulletin, 24, 213-216.

VALLEY, V., Broughton, R. (1983). The physiological (EEG) nature of drowsiness and its relation to performance deficits in narcoleptics. Electroencephalography \& Clinical Neurophysiology, 55, 243-251.

Vickers, D., Nettelbeck, T., * Willson, R. J. (1972). Perceptual indices of performance: The measurement of 'inspection time' and 'noise' in the visual system. Perception, 1, 263-295.

Wickens, C. D. (1984). Processing resources in attention. In R. Parasuraman \& D. R. Davies (Eds.), Varieties of attention (pp. 63-102). Orlando: Academic Press.

WilkInson, R. T. (1968), Sleep deprivation: Performance tests for partial and selective sleep deprivation. Progress in Clinical Psychology, $8,28-43$.

(Manuscript received June 21, 1990; revision accepted for publication October 7,1991 .) 